\title{
LOS TÉRMINOS LÉXICOS BÚHO, LECHUZA Y MOCHUELO EN LOS ATLAS LINGÜÍSTICOS ESPAÑOLES
}

\section{Erundina GARCERÁN INFANTES}

Universidad Nacional de Educación a Distancia (UNED)

\section{Introducción}

El objetivo principal de este trabajo es el análisis lingüístico de los datos léxicos que se contienen en los atlas lingüísticos españoles ALEANR IV, 461 y 462; ALEA II, 417 y ALEICan II, 3051, respecto de los términos búho, lechuza y mochuelo, en cuyo conocimiento y uso existen notables ambigüedades y diferencias entre los hablantes del español de las distintas regiones españolas.

El trabajo está precedido de una presentación teórica sobre los principales conceptos y datos que aporta la Geografía Lingüística, que justifica el marco y el método del análisis, puesto que la lengua hablada en zonas geográficas determinadas (Navarra, La Rioja, Andalucía e Islas Canarias) es el objeto formal del trabajo. El segundo apartado recoge el estudio de los datos lingüísticos de los atlas lingüísticos citados; y por último, como epílogo, presentamos el análisis de los resultados obtenidos respecto de la cala lingüística realizada sobre dichos ítems léxicos, en algunas localidades de la zona noroeste y capital de la provincia de Madrid.

\section{La Geografía Lingüística: su método}

Con el término de Geografía Lingüística se denomina tanto la disciplina lingüística que tiene como objeto el estudio de la lengua hablada en zonas geográficas determinadas, como el método de registro de los fenómenos lingüísticos que dicha ciencia emplea. Veamos a continuación, de modo sumario, los antecedentes históricos de la Geografía Lingüística.

A fines del siglo XIX, la concepción del dialecto, heredada de los gramáticos renacentistas, como variedad corrompida de la lengua culta, comenzó a sufrir una transformación al despertarse un interés progresivo por los estudios dialectales. Los primeros trabajos dialectológicos se realizaron bajo una perspectiva meramente descriptiva, pero centrados en la lengua hablada de zonas previamente delimitadas. El método más frecuentemente utilizado, en estos comienzos, fue el histórico-comparativo, el más estimado entre los lingüistas del momento. Sin embargo, al mismo tiempo, se empezó a utilizar como método propio, por considerarse más eficaz en el estudio de la lengua hablada, los registros de los fenómenos lingüísticos sobre representaciones cartográficas ${ }^{2}$. Dichos fenómenos lingüísticos habían sido recogidos previamente a través de encuestas directas y de modo oral, según un cuestionario establecido de antemano, y en un número de puntos geográficos de una misma zona. De es-

\footnotetext{
${ }^{1}$ Manuel Alvar, Atlas Lingüistico y Etnográfico de Andalucía (ALEA), I-IV, Universidad de Granada, 1961-1973. Con la colaboración de A. Llorente y G. Salvador. Manuel Alvar, Atlas Lingüístico y Etnográfico de las Islas Canarias (ALEICan), IIII, eds. del Cabildo Insular de Gran Canaria, 1975-1978. Manuel Alvar, Atlas Lingüistico y Etnográfico de Aragón, Navarra y Rioja (ALEARN), I-XII, Madrid- Zaragoza, 1979-1982. Con la colaboración de A. Llorente, T. Buesa y E. Alvar.

${ }^{2}$ Sobre las características de ambos métodos lingüísticos, Cf. Lingüística Románica, Madrid (U.N.E.D.), 1977, pp.56-57.
} 
ta manera, surgió como disciplina científica la denominada Geografía Lingüística a la que se identifica, desde sus comienzos, con su propio método.

En esta línea, una de las caracterizaciones más precisas de la Geografía Lingüística es la que hace Casado Velarde (1988: 24)3 cuando dice que la expresión geografía lingüística designa exclusivamente un método dialectológico y comparativo [...] que presupone el registro en mapas especiales de un número relativamente elevado de formas lingüísticas (fónicas, léxicas y gramaticales) comprobadas mediante encuesta directa y unitaria en una red de puntos de un territorio determinado. Los mapas lingüísticos elaborados según este método revelan, entre otras cosas, la conexión entre la historia lingüística y los factores geográficos o geopoliticos: permiten comprobar que las innovaciones en las lenguas proceden de ciertos centros y que su difusión se detiene en ciertos límites constituidos por ríos, montañas, fronteras políticas, administrativas o eclesiásticas; que la rapidez y amplitud de difusión depende del prestigio de los individuos y de los centros innovadores y de la intensidad y amplitud de las relaciones sociales y culturales.

El hecho de representar los fenómenos lingüísticos en forma de mapas geográficos tiene sus antecedentes en el trabajo del francés Bondelli Atlante Linguistique d'Europe, publicado en 1841. Por esa época, también el italiano Ascoli incluye un mapa lingüístico en sus Saggi Ladini. Entre estos precedentes hay que recordar a George Wenker, que en 1876 lleva a la práctica un método lingüístico muy similar al utilizado después por la Geografía Lingüística, aunque sólo atiende a fenómenos fonéticos. Sin embargo, es el francés-suizo Jules Gillièron, profesor de Dialectología en París, el que ostenta el título de padre de la Geografía Lingüística, puesto que con su Atlas Linguistique de la France (ALF), entre 1903 y 1910, señala el camino de los trabajos posteriores sobre atlas lingüísticos.

Los atlas lingüísticos han contribuido a enriquecer la lexicografía, aumentando considerablemente las formas lingüísticas de los diccionarios. Por citar un ejemplo, en el ALEA (Atlas Lingüístico y Etnográfico de Andalucía) se han recogido 600.000 formas en veinte años de trabajo, mientras que el Diccionario de la Real Academia Española (R.A.E.) actualmente contiene unos 85.000 vocablos. Los atlas nos ofrecen muchos significantes para un significado, como base para establecer las relaciones interdialectales y su distribución geográfica.

Después del ALF (Atlas Linguistique de la France) el número de atlas lingüísticos aumenta progresivamente, sobre todo en el campo de las lenguas romances. Entre los más significativos, en el área iberorrománica ${ }^{4}$ podemos destacar los siguientes: Antoni Griera (1923-1964): Atlas Linguistic de Catalunya; Antoni Griera (1964): Atlas Linguistic de Andorra; T. Navarro Tomás (1962): Atlas Lingüístico de la Península Ibérica (ALPI); Manuel Alvar (1961-1973): Atlas Lingüístico y Etnográfico de Andalucía (ALEA); Manuel Alvar (1975 y siguientes): Atlas Lingüístico y Etnográfico de las Islas Canarias (ALEICan); Manuel Alvar (1979-1982): Atlas Lingüístico y Etnográfico de Aragón, Navarra y La Rioja (ALEARN); C. García: Atlas Lingüístico Gallego (ALGA); Manuel Alvar (1995): Atlas Lingüístico y Etnográfico de Cantabria (ALEC); Manuel Alvar (1999): Atlas Lingüístico de Castilla y León ( $A L C y L)$. En proyecto existe un atlas nacional de Portugal y un atlas regional de Castilla-La Mancha. Y en Hispanoamérica existen los atlas de

\footnotetext{
${ }^{3}$ Manuel Casado Velarde, Lenguaje y Cultura, Madrid (Síntesis) 1988.

${ }^{4}$ Otros atlas lingüísticos, además de los mencionados, en Lingüistica Románica, Madrid (U.N.E.D.), 1977, pp.55-56.
} 
Puerto Rico, Chile y Colombia. Este último incorpora material sonoro en dos discos y consta de seis volúmenes. En proyecto están también los atlas de Costa Rica y Venezuela. En 1991 se publicó el cuestionario del Atlas Lingüístico y Etnográfico de Méjico, y es reciente la publicación del cuestionario del Atlas Nacional de Hispanoamérica, cuyos autores son Manuel Alvar y Antonio Quilis. Asimismo, Neddy A. Vigil y Garland D. Bills han publicado recientemente en la revista electrónica Círculo de Lingüística aplicada a la Comunicación 10, 2002, “El Atlas Lingüístico de Nuevo Méjico”.

\subsection{Aportaciones de la Geografía Lingüística}

La repercusión de la Geografía Lingüística ha sido considerable en todos los dominios de la lingüística, especialmente en la Dialectología. Su aportación teórica puede ser sintetizada en dos puntos: a) la defensa de la prioridad y la autonomía de la palabra respecto al sonido; y b) el reconocimiento del aspecto psicológico o espiritual del lenguaje, considerando que la acción del hablante influye esencialmente en su evolución.

La Geografía Lingüística ofrece la posibilidad de descubrir las conexiones culturales que subsisten en el lenguaje; así, basado en su método, Gerard Rohlfs ${ }^{5}$ descubre que los orígenes de la lengua griega que se habla en algunos lugares del sur de Italia se remontan a la época de la Magna Grecia y no a época bizantina, como se creía anteriormente. Con palabras de Rohlfs, de la Geografía Lingüística se pueden descubrir nuevos impulsos para la etimología. [...] cuando carecemos de fuentes históricas, la Geografía Lingüística nos ofrece a menudo la única posibilidad de descubrir viejas conexiones culturales.

La semántica también se aprovecha de la Geografía Lingüística. El estudio sincrónico de la lengua permite comparaciones entre las distintas etapas que son de gran eficacia para el estudio de fenómenos como la polisemia, la sinonimia y las neutralizaciones semánticas. Según Coseriu (1986) ${ }^{6}$, las lenguas tienen también una historia propia que sólo parcialmente se corresponde con la historia cultural de una determinada comunidad, y en esta historia también interviene la Geografía Lingüística, ayudando a conocer la historia de las palabras, las migraciones de las mismas, los neologismos, las destrucciones, los préstamos lingüísticos, las analogías, etc. Pero, en palabras de Coseriu (1986), las lenguas existen y se desarrollan no sólo, en virtud de las razones internas de equilibrio, como sistemas (relaciones estructurales), sino también y principalmente en relación con otros fenómenos del espíritu y de la sociedad. La lengua está íntimamente relacionada con la vida social, con la civilización, el arte, el desarrollo del pensamiento, la política, etc.; en una palabra, con toda la vida del hombre. Y como ya hemos dicho, la Geografía Lingüística es una gran impulsora y descubridora de este aspecto del lenguaje.

Por último, debemos reseñar el influjo que la Geografía Lingüística ha ejercido en el campo de los estudios teóricos del lenguaje, favoreciendo el nacimiento de corrientes como Worter und Sachen de Schuchardt y Meringer, la Onomasiología del suizo Ernest Tappolet, la Geología y Estratigrafía Lingüística y la Neolingüística o Lingüística espacial, entre otras 7 .

\footnotetext{
${ }^{5}$ Gerard Rohlfs, Estudios sobre el léxico románico, Madrid (Gredos) 1979.

${ }^{6}$ Eugenio Coseriu, Introducción a la Lingüistica, Madrid (Gredos), 1986.

${ }^{7}$ Véase Lingüística Románica, Madrid (U.N.E.D.), 1977, pp.57-60, sobre los citados movimientos.
} 


\section{Comentarios de los mapas lingüísticos}

A continuación comentaremos lingüísticamente los datos de los siguientes mapas: ALEARN IV, 462; ALEA II, 417 y ALEICan II, 305, correspondientes a los términos búho, mochuelo y lechuza, en las regiones de Aragón, La Rioja, Navarra, Andalucía y Canarias.

Como introducción a este estudio diremos que las clasificaciones de los seres de la naturaleza, establecidas por biólogos y naturalistas, presentan notables divergencias respecto de las consideradas y aceptadas por la mayoría de los hablantes de una lengua. En el caso que nos ocupa, existen creencias populares que asocian a búhos con mochuelos y lechuzas, y, aunque las tres pertenezcan al grupo de las aves rapaces nocturnas de la misma familia de las estrígidas, tienen entre sí notables diferencias biológicas. Así, el principal rasgo que caracteriza al búho es el grupo de penachos de plumas a modo de orejas o cuernos; el mochuelo, sin embargo, carece de estos penachos, de ahí su nombre (del latín mutilu, 'que le falta o carece de algo') ${ }^{8}$. La lechuza, además de carecer de dichas plumas, emite unos gruñidos parecidos a los del mochuelo, pero su principal característica es la costumbre de chupar el aceite que encuentra en los olivos o en las alcuzas, y esto es lo que probablemente originó su nombre vulgar.

\subsection{La denominación 'búho'}

La palabra búho procede del étimo latino būbo, -ōnis (del griego, bōas), su acusativo bubōne $(m)$ originó bubón, y por asimilación vocálica, al trasladarse el acento, bobón, que se generalizó en el aragonés. Según García de Diego (1981: 282) lo que ocurre en esta generación léxica es lo que él denomina una derivación regresiva, en la que las palabras se acortan por eliminación de un sufijo o una terminación ${ }^{9}$. En este caso, parece lógico pensar que la evolución de los términos búho y bubón tienen como origen las formas del nominativo y acusativo latinos, respectivamente.

\subsubsection{El mapa lingüístico ALEARN IV, 462}

Este mapa recoge las denominaciones del término castellano búho (bufo) y sus variantes fonéticas, en las zonas geográficas de Aragón, Navarra y La Rioja. Como se puede observar en la representación cartográfica, la forma más extendida es la castellana, y sólo en las zonas limítrofes se dan otras denominaciones como bubaro, bagueso, mochuelo, ontza (vasquismo), duget de nit (catalanismo).

\footnotetext{
${ }^{8}$ Cfr. Ramón Menéndez Pidal, Ramón, Manual de gramática histórica española, Madrid (Espasa-Calpe), 1989, p.118, y Vicente García de Diego, Gramática histórica española, Madrid (Gredos), 1951 (1981, $3^{\text {a }}$ edición corregida), p.37.

${ }^{9}$ Cfr. Vicente García de Diego, Gramática histórica del español, Madrid, Gredos, 1981, pág. 282. Este autor dice literalmente que "frente a la derivación normal en que una palabra se alarga por la agregación de uno o varios sufijos, se da la derivación regresiva, en que las palabras se acortan por eliminación de un supuesto sufijo, creyendo el hablante que así forma el nombre primitivo. El caso más notable es el del aumentativo en -ón. Se había supuesto que las formas en -o procedentes de nombres en -ón eran verdaderos nominativos; pero esta idea parece débilmente fundada. Es verdad que en la lengua culta hay verdaderos nominativos, como prefacio..., comparables al mayor número de formas francesas como confesse, de confessio; generace de geneatio; dedicace, de dedicatio...; pero no es admisible invocar al juego del nominativo y acusativo más que en los conocidos latinismos, y no en las formas vulgares hispanas, que no conocieron los dos casos de la declinación, sino exclusivamente el acusativo. La generalidad de los nombres en -ón, formados sobre tipos en -o, se supone que tendrán un positivo en -o. Los romances a veces han mantenido las dos formas del ponderativo y el positivo: bufone $>$ bufón, bufu $>$ buho". Véase también Ramón Menéndez Pidal, Manual de Gramática histórica española Madrid (Espasa-Calpe) 1989, p.209.
} 
Desde el punto de vista fonético, y en cuanto al consonantismo, tenemos que destacar lo siguiente:

1) la permanencia de la oclusiva bilabial sonora inicial en todas las realizaciones, $\mathrm{y}$

2) el predominio de la no-realización de la segunda consonante por pérdida de la aspiración, fenómeno que ocurre en las zonas de mayor influencia castellana como son Zaragoza, Logroño y algunos puntos de Logroño y Teruel. Sin embargo, hay una fuerte de contaminación catalana en Huesca y en algunas zonas del este de Teruel y Zaragoza. En estas zonas se realiza [bobón], [bubón] o [bobó], y en algún punto también [búgo]. Mientras que esta última variante es poco frecuente (Hu 601, Z 601) ${ }^{10}$ e incluso alterna con [bú|] en Na 501, la primera variante está muy extendida en la provincia de Huesca.

Quizás por influjo del castellano, en bufo [búpo], la segunda consonante al estar en posición intervocálica se fricatizó totalmente, y lo hizo parcialmente en [bobón] y [bubón]. Este influjo se notó en algunos límites castellanos con las provincias de Zaragoza (Z 201, 202, 400, 601), y Teruel (Te 202). El influjo de la fricatización de la oclusiva bilabial sonora intervocálica llega incluso a Na 309, aunque sin traslación del acento.

En cuanto a las vocales, la primera [ú] se mantiene por ser tónica en casi todo el territorio, alargándose incluso en muchos casos. Sólo en las zonas donde el acento se trasladó a la segunda vocal ha habido abertura de la primera vocal por asimilación con la [ó] tónica. En las zonas de influjo castellano, la segunda vocal ha tendido a abrirse, y en muchos casos, se ha perdido posteriormente. Habría que añadir, para explicar las variantes descritas, que quizás los pasos fonéticos de la forma búho fueron los siguientes: [būbo] (nominativo latino) > [búfo] (por fricatización de la [-b-] intervocálica) > [búo] (por aspiración y posterior pérdida de la segunda consonante). Mientras que de su acusativo se originaron las demás denominaciones: [bubōnem] > [bubón], [bu甲ón] y [bobón].

Para abordar el estudio léxico semántico debemos recurrir, en primer lugar, a la definición que nos da el Diccionario de la Lengua Española de la R.A.E. ${ }^{11}$ del término búho: (del latín bufus, dialectal de bubos, - ōnis) $m$. Ave rapaz nocturna, indígena de España, de unos 40 centímetros de altura, de color mezclado de rojo y negro, calzada de plumas, con el pico corvo, los ojos grandes y colocados en la parte anterior de la cabeza, sobre la cual tiene unas plumas alzadas que figuran orejas. Respecto al mismo término, las Enciclopedias Sopena ${ }^{12}$ nos dicen: 1) (del latín bubo), m. Ave rapaz nocturna, indígena de España, de cabeza grande, aplanada y con dos mechones de plumas que se asemejan a las orejas de un gato, pico corvo, ojos grandes y cuerpo grueso y robusto, de color mezclado de rojo y negro; y 2) Nombre de ave rapiña nocturna de la familia de las estrígidas, cabeza y ojos grandes dirigidos hacia adelante. Pico y garras fuertes. Vuelo silencioso. Llamativos penachos en la cabeza a

\footnotetext{
${ }^{10}$ Para facilitar las anotaciones lingüísticas en los mapas, se dividen las provincias geográficas (representadas por sus iniciales) en 6 zonas, y se enumeran las zonas encuestadas del siguiente modo: a partir de 100 (la mitad noroeste), de 200 (la mitad noreste), de 300 (la mitad centro-oeste), de 400 (la mitad centro-este) de 500 (la mitad suroeste), y a partir de 600 (la mitad sureste). En cada una de las zonas, la numeración se realiza de izquierda a derecha y de norte a sur.

${ }^{11}$ Cfr. Diccionario de la Lengua Española de la Real Academia Española, Madrid (Espasa-Calpe), 21 a edición, 1994; Tomo I, p.331.

${ }_{12}$ Enciclopedia Sopena (2 tomos): Barcelona, 1926; y Enciclopedia Universal Sopena (9 tomos), Barcelona, 1964.
} 
modo de 'cuernos'. Búho real: la mayor de las estrígidas de Europa, mide 1,70 m., caza al amanecer, tiene costumbres solitarias, grandes ojos de color naranja, duerme en rocas hundidas o troncos huecos. Sin embargo, la palabra búho no parece provenir de ninguna de las características que enumeran los diccionarios, sino de la lexicalización del sonido que emite esta ave rapaz, parecidos a $b u$, uh, por lo que búho se considera, desde su origen, un término onomatopéyico.

En Na 206, se denomina [baßwéso] a otro ave rapaz muy parecida al búho, pero que se diferencian en el sonido que emiten, siendo el de ésta más largo que el del búho.

En $\mathrm{Hu}$ 102, junto a bavueso o babueso se utiliza avetoro [aßetóro], variedad de búho, también con cabeza gorda y dos mechones de plumas a modo de orejas. El nombre es un compuesto metafórico, el primer término, ave, hace referencia a la especie a la que pertenece, y el segundo término, toro, expresa su parecido con este animal debido a los dos mechones en forma de cuernos que caracterizan al búho. Idéntica motivación subyace en la denominación cabrero [kaßréro], que se encuentra en $\mathrm{Hu} 103$ y 108, es decir, su parecido con las cabras por los cuernos de pluma. Aunque tanto las cabras como los toros se identifican por sus cuernos, quizás tenga el búho en común con el toro, además de los 'cuernos', el gruñido, parecido al mugido del toro тиии.

En esta línea, se puede justificar la motivación de la forma cornuillo [kornwíyo] usado en Lo 605, junto a búho. De nuevo se hace referencia a los mechones de pluma de la cabeza del búho, aunque esta vez se utiliza el sustantivo castellano cuerno para componer un diminutivo yeísta con el sufijo -illo.

La denominación de babieca [baßjéka] que existe en $\mathrm{Hu} 202$ también resulta un término metafórico puesto que babieca era el nombre del caballo del Cid, y quizás se utiliza por el parecido del relincho del caballo con el gruñido del búho.

La denominación ontza [ónӨsa] que se registra en Na 102 y 104, es un vasquismo, que alterna con la forma castellana búho [búo] en dicha zona. Del mismo modo el sintagma nominal duget de nit, 'señor de la noche'13, que se utiliza en Z 605, frontera con Cataluña, es un contagio del catalán.

En Te 402 y 405 existe la denominación carabo para designar al búho. El carabo es un ave rapaz nocturna parecida a la lechuza aunque algo más grande. El término $c a-$ rabo proviene del árabe carab. La realización fonética en dichos puntos geográficos son [kárbo] y [karbú], respectivamente.

Observamos que en Na 100 y 103 existe un contagio del vasco, por ser zona limítrofe, puesto que se usan los términos untza y ontza tanto para designar al búho como a la lechuza.

Según el citado mapa lingüístico, aparte de las variedades comentadas, la voz castellana búho es término único en $\mathrm{Bu}, \mathrm{Vi}$, Lo, Na, So, Gu, Cu y V. En Z, sólo existe la excepción del término chuta, deturpación o deformación de chupa, motivada por la costumbre del animal de chupar el aceite, por lo que en algunos lugares se le llama

\footnotetext{
${ }^{13}$ Análogo a la expresión catalana duget de nit es el término francés duc con el que también se denomina al búho, incluso grand duc es una variedad de búho.
} 
también oliba (Te, 307, 504 y 601) y olipa (Te 405). En Hu se documenta también coruja (Hu 204), meuca (Hu 205 y 404) chuta (Hu 401, 404, 407, 408, 600 y 603) y chut (Hu 402). En Na 202 y Te 503 se recoge la deturpación lechuga.

En el ALEARN IV, 461, se han recogido algunas variantes fonéticas de la denominación búho, debidas generalmente al dialectalismo de las zonas en que se dan. Así, junto a la forma castellana, existen las formas [bubáxo], 'búho grande', en Lo 601, y la forma [bußarílo], 'búho pequeño', en Lo 603. El sufijo -aja se usa como aumentantivo, mientras que el sufijo -illo se utiliza para formar el diminutivo. En la composición de estos derivados se utiliza la raíz léxica del acusativo latino, bub-, por tener mayor cuerpo fónico que la del nominativo. En estos casos, también se produce una fricatización de la segunda consonante, por las mismas causas antes explicadas, por ejemplo, en Na 301, 302 y 306 existen realizaciones como [bußáxo], [bußářo], [bußáxo], respectivamente, que no alternan con la forma castellana.

\subsection{La denominación 'lechuza'}

Acudimos, en primer lugar, a las definiciones de los diccionarios respecto del término que nos ocupa. Así, en el Diccionario de la Lengua Española de la R.A.E. ${ }^{14}$ se dice: Ave rapaz nocturna de unos 35 centímetros de longitud desde lo alto de la cabeza hasta la extremidad de la cola, y aproximadamente el doble de envergadura, con plumaje muy suave, amarillento, pintado de blanco, gris y negro en las partes superiores, blanco de nieve en el pecho, vientre, patas y cara; cabeza redonda, pico corto y encorvado en la punta, ojos grandes, brillantes y de iris amarillo, cara circular, cola ancha y corta y uñas negras. Es frecuente en España, resopla con fuerza cuando está parada, y da un graznido estridente y lúgubre cuando vuela. Se alimenta ordinariamente de insectos y de pequeños mamíferos roedores. El Diccionario Sopena ${ }^{15}$ añade: ...y también sorbe el aceite si lo halla cuando está hambrienta.

El término castellano lechuza procede con toda probabilidad de la característica de chupar de este animal. Y puesto que lo que generalmente es materia del chupar suele ser la leche materna, para los recién nacidos y crías animales, se ha tomado la raíz semántica del término leche ${ }^{16}$ para designar al animal que se caracteriza por la acción de chupar. A este sustantivo se le añade, para evitar la homonimia y aludiendo al robo engañoso del aceite por parte del animal, el sufijo despectivo -uza (del latín -uceu $)^{17}$. Este desprecio popular se puede deber al hecho de robar traicioneramente sus presas y su comida, y probablemente, es la causa de ser considerado tradicionalmente, igual que el búho, pájaro de mala suerte o de mal agüero ${ }^{18}$.

\footnotetext{
${ }^{14}$ Cf. nota 11.

${ }^{15}$ Cf. nota 12.

${ }^{16}$ Recordemos que, desde el punto de vista fonético, la derivación de leche a partir del étimo latino lacte(m) es como sigue: por vocalización de la consonante $c$ en el grupo $c t$ se origina laite, posteriormente, por influjo de esta yod, se palataliza la $t>$ $\mathrm{ch} / \check{\mathrm{s}} /$ de donde se deriva leche, esta palatalización se atribuye al sustrato celta, así como el cierre de la á tónica $>e$. Cfr. Manuel Ariza Viguera, Manual de fonología histórica del español, Madrid (Síntesis) 1989, pp.118-120.

${ }^{17}$ La pregunta del cuestionario que sirvió de base para el ALEICan II, 305, contenía la siguiente descripción del animal: 'pájaro grandecito que está en la torre de las iglesias y bebe el aceite de las lamparillas'. Cfr. Vicente García de Diego, Gramática histórica española, Madrid (Gredos), 1951 (3 $3^{\text {a }}$ ed. corregida, 1981), p.272, sobre el sufijo despectivo -uza.

${ }^{18}$ Entre otras citas literarias, que documentan este dato, se encuentra la Fábula de Píramo y Tisbe de Góngora, donde el búho aparece presagiando la muerte de los dos amantes, al final del poema.
} 


\subsubsection{El mapa lingüístico ALEICan II, 305}

Este mapa representa la distribución geográfica de las distintas denominaciones que se dan en Canarias al término castellano lechuza (Athene noctua). Pero no representa las variaciones fonéticas, sino solamente las familias léxicas.

Como se observa en el citado mapa, el término castellano está muy extendido en las cuatro islas occidentales: La Palma, Tenerife, Gomera y Hierro. Sin embargo, se alterna con la denominación coruja en las tres islas restantes: Gran Canaria, Fuerteventura y Lanzarote. En esta última se identifica al animal sobre todo por el sonido que emite, parecido al del llanto de un niño ( $\mathrm{Lz} 2$ ) o una persona ( $\mathrm{Lz} 3$ ).

En Fuerteventura perviven las formas lechuza, coruja y las aves [loӨ-ábë]. El término general de las aves, junto con el de lechuza, se da también en Tf 5 [los-ábëh] y en Go 3 [los-á:bëh]. En Go 4, además de lechuza, se da un dialectismo que significa 'las aves' [aßaỹú:yë]. En La Palma (LP 3), junto con lechuza, pervive el término [aßikán] que hace referencia al modo de volar: 'volar en picado'.

En Fuerteventura ( $\mathrm{Fv} 30)$ se realiza la forma castellana con cambio de género lechuzo [leĉú:so], y lo mismo sucede en GC 2, 3, 10, 11, 12, 20 y 40; en ambas provincias con el significado de 'mochuelo', debido quizás al tamaño más pequeño del mochuelo respecto de la lechuza. Como es sabido, la distinción de género suele indicar, en muchos casos, diferencia de tamaño, por ejemplo, cesto (más pequeño), cesta (más grande).

En conclusión, podemos generalizar diciendo que existe un predominio del término castellano lechuza para denominar el conjunto de las aves rapaces nocturnas de la misma familia y de características semejantes como son el búho, la lechuza y el mochuelo. La segunda denominación más frecuente es la de coruja, que es un lusismo que también se usa en algunos puntos de Andalucía occidental fronterizos con Portugal, y desde donde se trasladaron a las Islas Canarias, al mismo tiempo que las formas castellanas. Recordemos que este lusismo también se utiliza de forma aislada en la provincia de Huesca (Hu 204), zona muy conservadora y de dialectismos. Así, en Canarias, dicho lusismo prevalece, alternando con la forma castellana, en el oriente canario, más conservador por tener menos influencia exterior, debido a su mayor aislamiento geográfico. Aunque, en general, los dialectismos se dan de manera muy aislada y tienden a sustituirse por las formas castellanas.

En algunos puntos se resuelve el polimorfismo introduciendo el término genérico castellano las aves, que es la tercera denominación más frecuente que se ha sido registrada. Al ser una palabra demasiado genérica, se tiende a singularizarla añadiéndole el artículo. Y el uso del plural puede tener su explicación en la intención de obtener una mayor expresividad, aumentar el cuerpo fónico de la palabra ave, o en el objetivo de extender el campo semántico y, al mismo tiempo, evitar la homonimia con el singular.

\subsubsection{El mapa lingüístico ALEA II, 417}

Con respecto al término lechuza, en la zona andaluza, se utiliza de forma generalizada la forma castellana, y sólo en algunos puntos aislados de Huelva y Córdoba (Hu 100, 101, 102, 200, y Co 102, 200) aparece el occidentalismo (lusismo) coruja, y es 
precisamente del occidente andaluz desde donde pasará a Canarias durante la colonización de las Islas, donde se conservará alternando con la forma castellana que se va imponiendo en el oriente isleño.

Con cambio de género, muestra de la expresividad del habla andaluza, se usa lechuzo [leĉúӨo], en Jaén (J 202).

\subsection{La denominación 'mochuelo'}

Mochuelo es término castellano que se refiere a un ave rapaz de la misma familia que el búho y con características muy parecidas, pero sin los penachos típicos de éste. La misma palabra mochuelo proviene del latín mutilu 'que le falta algo', que da origen a $m o c h o^{19}$, por palatalización del grupo $t i$, a cuya raíz se le ha añadido el sufijo -uelo que posee un significado despectivo, igual que el sufijo -uza del término lechuza, y probablemente por el mismo motivo. Popularmente, el mochuelo y el búho están muy relacionados.

En el Diccionario de la Lengua Española de la R.A.E. ${ }^{20}$ se dice lo siguiente de la palabra mochuelo: Ave rapaz nocturna, de unos dos decímetros desde lo alto de la cabeza hasta la extremidad de la cola, y medio metro aproximadamente de envergadura, con plumaje muy suave, de color leonado, con pintas pardas en las partes superiores, y amarillento claro con manchas alargadas grises en el pecho y vientre; cuerpo erguido, cabeza redonda, pico corto y encorvado, ojos grandes de iris amarillo, cara circular, alas redondeadas, cola corta y tarsos $y$ dedos cubiertos de plumas blanquecinas y sedosas. Es común en España y se alimenta ordinariamente de roedores y reptiles.

En la Enciclopedia Sopena ${ }^{21}$ se da la siguiente definición de mochuelo: (de mocho), ave rapaz nocturna, de plumaje muy suave, de color leonado, picocorvo, ojos muy grandes y redondos, vive de roedores pequeños y reptiles. Es común en España. Y en la Enciclopedia Universal Sopena se dice: ave nocturna de rapiña. De la familia de las estrígidas. En Europa. Tamaño pequeño y plumaje gris oscuro, rojizo en el dorso y más claro en el vientre con pintas blanquecinas. Caza en las primeras horas de la mañana y de la noche. Come pequeños roedores, insectos y reptiles. Emite gritos monótonos y agudos cuando se le molesta, que le han dado fama de pájaro de mal agüero, igual que ocurre con el búho, y que está bien documentado en la literatura ${ }^{22}$.

\subsubsection{El mapa lingüístico ALEA II, 417}

Este mapa representa las denominaciones de las formas castellanas lechuza y mochuelo en la zona geográfica de Andalucía, aunque aquí nos centraremos solamente en la palabra mochuelo.

En esta zona, y por la cercanía con Castilla, se utiliza la forma castellana mochuelo, aunque se recogen variantes fonéticas como machuelo, quizás por analogía con macho, en puntos de Huelva, Sevilla y Granada (H 101, 200, 300, 302, Se 300, y Gr 306), michuelo (Se 402, 501), morchuelo (J 308, Al 508), muchuelo (Co 101, 603 y J 103). El cambio de género, mochuela, por analogía con mozuela ${ }^{23}$, se registra en Se 401, Ma 300,

\footnotetext{
${ }^{19}$ Cf. nota 8.

${ }^{20}$ Cf. nota 11.

${ }^{21}$ Cfr. nota 12.

${ }^{22} \mathrm{Cfr}$. nota 18

${ }^{23}$ Un cierto machismo, especialmente cultivado en Andalucía, pudiera ser la motivación léxica de este cambio de género.
} 
J 200, Al 503. En suma, la motivación de estas formas parece encontrarse en la analogía fonética y en el afán de una mayor expresividad lingüística.

La forma regresiva mocho se encuentra en uso en puntos de la provincia de Huelva (H 303 y 501), probablemente por contaminación del portugués que la utiliza frecuentemente.

Se recogen también dos variantes léxicas como son las formas cuco (J 400 y 600), motivada por el parecido de ambos animales en el sonido que emiten; y juanillo [gwaníyo] en Ma 102, de carácter humorístico, y probablemente originado por la analogía existente en el comportamiento de este animal y en el del personaje literario Don Juan: conseguir sus presas robando y engañando. Es curioso observar como en el mapa lingüístico $A L E A R N I V, 461$, también a la urraca, y quizás por el mismo motivo, se le llama juana en un punto de Navarra ( $\mathrm{Na} 601$ ), alternando con el término castellano, y juanaza en Logroño (Lo 500), donde el sufijo -aza refuerza el valor despectivo del término.

\subsubsection{El mapa lingüístico ALEARN IV, 461}

Aunque este mapa lingüístico recoge las denominaciones del término castellano chotocabras, sin embargo, en sus notas se registra también algunos resultados respecto de la forma mochuelo, en la misma zona geográfica de Aragón, La Rioja y Navarra, y zonas limítrofes, que pasamos a comentar a continuación.

Se recogen las siguientes variantes, alternando con la forma castellana, que pueden tener las mismas motivaciones fonéticas que en Andalucía: morchullo (Lo 604), machuelo (Na 201), mozuelo (Bu 400), muchuelo (Z 201, 604). Mochó, que aparece en Hu 406 , es la forma regresiva con traslación del acento por influencia catalana. Alternando con mochuelo, que es la forma más extendida, se encuentra búho en $\mathrm{Na} 202,203$, 204 y Z 200. En Na 201 y Hu 402, a la chotocabra, se le dan los mismos nombres que a la lechuza: lluc y meuca, respectivamente.

Puede esclarecer en algo las motivaciones de estas denominaciones y la confusión de las formas búho y mochuelo, la siguiente cita de Alvar ${ }^{24}$ referente al mapa lingüístico ALEARN I, 43: En Lo 100, se le da el nombre de mocho al 'espantapájaros', pero mocho es la forma positiva de donde salió el castellano mochuelo, de apariencia diminuta. Creo que no se separan las creencias populares que asocian búhos y mochuelos, pues incluso el nombre de éste procede de una comparación con aquél: no tiene plumas en la cabeza y el búho sí. Pero el búho es ave rapaz temida por otras muchas, lo que explicaría que se designe como 'espantapájaros' un mocho del que ha desaparecido la idea empequeñecedora, pero no su relación con el búho. Por otra parte no olvidemos el 'coco' de los temores infantiles, tantas y tantas veces representado por bu, bute y formas emparentadas con la voz castellana búho.

\subsection{Conclusiones}

En primer lugar, debemos subrayar la extraordinaria riqueza del léxico español, patente a la luz del presente estudio.

En la formación del léxico, además de los préstamos lingüísticos recibidos del latín y de otras lenguas, el español, como todas las lenguas, ha desarrollado los recur-

\footnotetext{
${ }^{24}$ Cfr. Manuel Alvar, Estudios de Geografia Lingüistica, Madrid (Paraninfo) 1990.
} 
sos necesarios para denominar las realidades existentes con nuevos términos; y una de las principales fuentes de la creación léxica es, sin duda, la cultura popular.

A continuación, exponemos de forma ordenada, las principales motivaciones que parecen configurar el léxico estudiado:

1) Motivaciones onomatopéyicas: son determinantes en los nombres de aves; así, la palabra búho ya constituía en latín (būbo, -ōnis) una forma onomatopéyica, es decir, que imitaba el ruido del ave rapaz nocturna, y esta motivación se mantiene en la forma castellana. La onomatopeya se acentúa aún más en realizaciones como [bú] o [bú:o], y varía en [bobón], [bubón]. La forma cuco, responde a la misma motivación.

2) Motivaciones visuales: esta motivación determinó la forma latina mutìlu, 'que le falta algo', y da origen a la evolución romance mocho. Son representativas también de este tipo de motivación las denominaciones del término castellano búho: avetoro, cabrero, cornuillo, carabo; todas ellas hacen referencia a los penachos de plumas, a modo de cuernos, que caracterizan al búho. Así mismo, bubarro y buvarillo son denominaciones del búho, motivadas por el tamaño, grande o pequeño respectivamente, del ave.

3) Motivaciones prosopopéyicas: este tipo de motivación, cargada de humorismo, es evidente en la forma juanillo con la que se denomina al mochuelo en algún punto de la provincia de Málaga. Así también, las denominaciones juana y juanaza de la urraca, en Navarra y Logroño. El origen de estas denominaciones posiblemente haya que encontrarlo en la relación que existe entre las aves rapaces nocturnas y el personaje literario de Don Juan, muy integrado en la cultura popular: lograr sus objetivos o sus presas engañando y robando.

4) Motivaciones etológicas: las diversas costumbres del animal han originado las formas léxicas de oliva y olipa (la lechuza chupa el aceite), el dialectalismo canario avicar ('vuelo en picado'). Probablemente, la forma babieca responda tanto a motivaciones visuales, por la semejanza de los penachos del búho con las orejas del caballo, como a motivaciones etológicas, por la semejanza del ruido que emite el ave con el relincho del caballo. La forma vulgar de lechuza (Athene noctua) ${ }^{25}$ puede haberse originado también por motivación etológica, basada en la costumbre del animal de chupar el aceite relacionada con el hecho paradigmático de chupar la leche materna por el niño y la cría animal. El sufijo despectivo -uza tendrá su origen en el desprecio que provoca el hecho de que el animal roba el aceite que chupa. El sufijo despectivo -uelo de mochuelo parece tener idéntica motivación. Éste parece ser también el origen de las creencias populares acerca del 'mal agüero' que se le atribuye a la presencia de estas aves.

En segundo lugar, debemos señalar también como conclusión que, a pesar del poliformismo existente en las denominaciones de estos animales, predomina el uso de los términos castellanos búho, lechuza y mochuelo en Aragón, Navarra y La Rioja; mientras que tanto en Andalucía como en Canarias no se usa, aunque se conozca, la forma léxica búho, lo que parece deberse a la existencia de una mayor expresividad

\footnotetext{
${ }^{25}$ Probablemente el nombre científico de la lechuza (Athene noctua) tenga su causa tanto en la nocturnidad de las costumbres de este animal, como en la zona donde se identificara el animal originariamente. Sin embargo, una vez más, se demuestra la brecha existente entre las denominaciones léxicas cultas y las populares, así como la imposición del uso de la lengua hablada.
} 
fonética de las formas lechuza y mochuelo, registradas con la mayor frecuencia en Andalucía de donde pasan a las Islas Canarias.

En tercer lugar, debemos concluir que la confusión que se registra, en todas las zonas estudiadas, entre las denominaciones y el conocimiento de los referentes reales, es decir, entre estos tres tipos de aves rapaces nocturnas, parece deberse a dos factores: el gran parecido de las aves entre sí y al desconocimiento de la realidad natural, esto es de estos animales, probablemente por la concentración de la vida en las ciudades y la falta de contacto con la naturaleza. La lechuza y el mochuelo son objeto de mayor confusión debido a que se parecen más entre sí por la carencia de los penachos, característicos del búho. A ambas aves se les llama untza, ontza, onz, unztiquilla, en la frontera vasco-navarra por contagio del vasco. El caso más representativo de esta confusión se ha registrado en la localidad de Egozcua (Na 202), donde al mochuelo se le llama búho, al búho, mochuelo, a la chotocabra se le denomina también búho, y a la lechuza se le da el nombre de lechuga por deturpación del término castellano.

Este confusionismo parece ser la causa de la tendencia a la generalización de las denominaciones que se registra en todas las zonas estudiadas. En Aragón, Navarra y La Rioja se utiliza el término generalizado búho para nombrar las tres aves. En Canarias se tiende a generalizar las denominaciones con el término lechuza. Por último, en Andalucía, es la palabra mochuelo la más generalizada.

Las analogías fonéticas se registran sólo en el uso de la forma lingüística mochuelo; y esto ocurre en zonas concretas de los tres mapas estudiados. Las formas originadas más usadas son: morchullo, muchuelo, michuelo, machuelo, mozuelo. Las motivaciones de estas formas léxicas hay que buscarlas en la fuerza expresiva de la lengua hablada, en general.

Esta búsqueda continua de expresividad de la lengua hablada, más marcada en las zonas del sur, parece ser también la causa que ha motivado las formas lechuzo, en Canarias, y mochuela, en Andalucía, en las que existe un cambio de género respecto de los términos castellanos correspondientes. La relación lingüística entre Andalucía y Canarias es lógica puesto que fue desde Andalucía occidental desde donde partió la colonización de las Islas Canarias.

Por último, hay que destacar la contaminación lingüística que se registra en los límites fronterizos por influencia de los adstratos o lenguas en contacto. Así, en la frontera vasco-navarra se han registrado las formas léxicas antes señaladas, por contagio del vasco. En la provincia de Huesca y en su límite catalán existe una gran influencia de la lengua catalana en las formas olluc, meuca, meuca, chuza, muzól, musol, chuta, para designar a la lechuza y al mochuelo. También en la frontera catalana de la provincia de Zaragoza existe la influencia del catalán en los términos duget de nit, bobón, bubón, bobó, carbó, para denominar el búho. Hay que señalar también el lusismo coruja que se ha registrado en la frontera de Andalucía occidental con Portugal, desde donde pasa a Canarias, cuyas islas orientales, más cerradas a los influjos externos, la conservan. Por último, hay que destacar el contagio dialectal de los términos canarios aveyuya y avicar. 


\section{Encuesta en Madrid capital y la zona noroeste provincial}

En esta tercera y última parte del trabajo presentamos una encuesta sobre el término castellano búho, en la zona noroeste y capital de la provincia de Madrid. Más que una encuesta en toda regla, hemos tratado de hacer una cala léxica en una zona precisa y sobre un ítem léxico también determinado, que más bien representa un experimento sobre una encuesta. Los elementos de la misma son los siguientes.

\subsection{Zonas geográficas}

Las localidades en las que hemos realizado la encuesta son, además de Madrid capital (M 1), los siguientes pueblos del noroeste de la provincia de Madrid: Pozuelo de Alarcón (M 2), Boadilla del Monte (M 3), Majadahonda (M 4), Las Rozas de Madrid (M 5). Todas estas zonas poseen un gran porcentaje de población inmigrante, española y extranjera, por lo que nos hemos dirigido únicamente a personas de cada zona.

\subsection{El cuestionario}

El procedimiento utilizado ha sido el de solicitar de los encuestados contestaciones individuales, de forma oral y escrita, a las preguntas de un cuestionario, fijado de antemano. El contenido de dicho cuestionario, además de los datos del encuestado, es el siguiente: 1) ¿Cómo se llama el ave de rapiña nocturna, con dos penachos de plumas a modo de cuernos, garras fuertes, pico corvo, ojos grandes hacia adelante, emite ruidos y ronquidos, y chupa el aceite de las olivas? 2) ¿Conoces algún otro nombre de este animal? 3) ¿Conoces otra(s) ave(s) de rapiña nocturna parecida(s) a este animal? Si es así, di su(s) nombre(s) y su(s) característica(s) principales 4) ¿Conoces algún dicho popular o refrán en relación con este (estos) animal(es)?.

\subsection{Los informantes}

Hemos solicitado las contestaciones de representantes de ambos sexos y de tres generaciones. Es decir, en cada una de las cinco localidades han sido encuestados 50 hombres y 50 mujeres de cada una de las tres generaciones: la primera compuesta por personas de edades entre 20 y 35 años; la segunda generación, entre 36 y 55 años; y la tercera, de 56 a 70 años. Un total de 150 mujeres y 150 varones en cada una de las cinco zonas, que componen un total de 1.500 personas. En ningún caso nos hemos dirigido a matrimonios para evitar la contaminación lingüística.

\subsection{Resultados de la encuesta}

El cuadro siguiente recoge los resultados obtenidos sobre las denominaciones del búho, por parámetros de zona, generación y sexo: 


\begin{tabular}{|c|c|c|c|c|c|c|}
\hline & Madrid & Pozuelo & Boadilla & Majadah. & Rozas \\
\hline $1^{\text {a gen. }}$ & $\mathrm{V}$ & $\begin{array}{c}\text { búho } \\
\text { lechuza }\end{array}$ & $\begin{array}{c}\text { búho } \\
\text { lechuza* }\end{array}$ & búho & búho & búho \\
\hline $2^{\text {a gen. }}$ & $\mathrm{V}$ & búho & $\begin{array}{c}\text { lechuza } \\
\text { búho }\end{array}$ & $\begin{array}{c}\text { mochuelo } \\
\text { lechuza }\end{array}$ & búho & búho \\
\hline & $\mathrm{M}$ & búho & búho & lechuza & búho & búho \\
\hline $3^{\text {a gen. }}$ & $\mathrm{V}$ & $\begin{array}{c}\text { lechuza } \\
\text { mochuelo }\end{array}$ & $\begin{array}{c}\text { mochuelo } \\
\text { búho }\end{array}$ & $\begin{array}{c}\text { lechuza } \\
\text { búho }\end{array}$ & búho & búho \\
\hline & $\mathrm{M}$ & $\begin{array}{c}\text { lechuza } \\
\text { mochuelo }\end{array}$ & $\begin{array}{c}\text { mochuelo } \\
\text { búho }\end{array}$ & $\begin{array}{c}\text { mochuelo } \\
\text { lechuza }\end{array}$ & búho & búho \\
\hline
\end{tabular}

$\mathrm{V}=$ varones $\quad \mathrm{M}=$ mujeres

*Lechucear: 'picar la comida de la nevera'.

\# Parece una lechuza: 'se dice de quien moja pan en salsas aceitosas'.

a) resultados fonéticos:

Las realizaciones fonéticas se han adecuado en todos los casos a la norma castellana: [búo], [moĉuélo], [leĉúӨa].

b) resultados morfológicos:

No se han dado casos de cambio de género ni utilización del plural. Se puede decir que, también en este aspecto, los resultados han respectado la norma castellana.

c) resultados léxicos:

El 85\% de los encuestados ha usado la denominación búho, mientras que el 30\% ha contestado con el término lechuza, y el 20\% con la palabra mochuelo. En suma, la forma más extendida es la de búho.

Por generaciones, todas las personas de la primera generación han dicho búho, entre ellos, sólo las mujeres de Madrid distinguen y usan correctamente las denominaciones búho y lechuza, mientras que en los hombres existe algo de confusionismo. En general, el término mochuelo no se distingue de los otros y, por tanto no se usa, existiendo una clara tendencia a denominar búho a cualquier ave rapaz de la familia de las estrígidas. Entre las mujeres de Madrid se conoce el término vulgar lechucear, 'picar la comida de la nevera'.

También en la segunda generación existe la tendencia a denominar búho a las demás aves rapaces. Sólo en Pozuelo se conoce la distinción entre mochuelo, lechuza y búho. Entre los hombres de Boadilla también se conoce la diferencia entre búho y lechuza. Tanto Pozuelo como Boadilla son las dos localidades más rurales de las encuestadas.

En la tercera generación, el $60 \%$ de los encuestados conoce y distingue las tres denominaciones; el 30\% conoce los nombres pero las confunde y tiende a la denominación generalizada búho. Los hombres y mujeres de Madrid son los que han dado una información más precisa de cada uno de los nombres y las aves rapaces; conocí- 
an la costumbre de chupar el aceite de la lechuza, y el dicho popular pareces una lechuza, 'se dice del que moja pan en salsas aceitosas'.

\subsection{Conclusiones}

Podemos interpretar los resultados expuestos de la siguiente manera:

1. La fonética y la morfología se ajustan totalmente a la norma castellana.

2. Se usan términos castellanos sin ninguna contaminación ni dialectismo, puesto que Madrid se ubica entre las dos Castillas.

3. Predomina el uso de la forma búho, que se tiende a generalizar progresivamente como denominación de todas las aves rapaces de la misma familia. Dicha generalización, debida al desconocimiento progresivo de la naturaleza, resulta muy evidente en la primera generación, un poco menos patente, en la segunda, y mucho menos evidente, en la tercera generación.

4. El término léxico lechuza es más conocido que el de mochuelo, que está prácticamente perdido en la primera generación. Así el dicho popular cada mochuelo a su olivo no es conocido por los encuestados de la primera generación.

5. No hay diferencias notables entre las respuestas de los distintos sexos.

6. Los encuestados de Madrid capital están más informados que los de las demás localidades donde se ha realizado el cuestionario, pero este hecho, en la mayoría de los casos, no es debido al conocimiento de la realidad referente sino a los conocimientos adquiridos por otros medios (libros, cine, televisión, etc.). Los de la tercera generación han tenido mayor contacto con las realidades naturales que los demás informantes.

7. En cuanto a las localidades circundantes a la capital, en las más urbanas, como Majadahonda y Las Rozas, se tiende en mayor grado a la generalización de la denominación búho y no se conoce la distinción real entre las aves rapaces; sin embargo, en los pueblos con mayor área rural, como Pozuelo y Boadilla, exceptuando la primera generación, distinguen, aunque con algo de confusionismo, sobre todo al mochuelo, como 'búho más pequeño', de las otras dos aves, porque en la zona ha existido o existe dicho animal.

8. El desconocimiento progresivo de la naturaleza animal probablemente sea la causa de la pérdida de las connotaciones de "mal agüero" que se habían venido predicando tradicionalmente de estas aves rapaces, y de las características de "buena suerte" con las que actualmente se definen. 